University of Nebraska - Lincoln

DigitalCommons@University of Nebraska - Lincoln

U.S. Department of Veterans Affairs Staff

Publications

U.S. Department of Veterans Affairs

$10-2003$

\title{
Do physicians examine patients in contact isolation less frequently? A brief report
}

\author{
Sanjay Saint \\ Ann Arbor Department of Veterans Affairs Medical Center, saint@umich.edu \\ Leigh Ann Higgins \\ Virginia Commonwealth University \\ Brahmajee K. Nallamothu \\ University of Michigan, bnallamo@umich.edu \\ Carol Chenoweth \\ University of Michigan, cchenow@med.umich.edu
}

Follow this and additional works at: https://digitalcommons.unl.edu/veterans

Saint, Sanjay; Higgins, Leigh Ann; Nallamothu, Brahmajee K.; and Chenoweth, Carol, "Do physicians examine patients in contact isolation less frequently? A brief report" (2003). U.S. Department of Veterans Affairs Staff Publications. 71.

https://digitalcommons.unl.edu/veterans/71

This Article is brought to you for free and open access by the U.S. Department of Veterans Affairs at DigitalCommons@University of Nebraska - Lincoln. It has been accepted for inclusion in U.S. Department of Veterans Affairs Staff Publications by an authorized administrator of DigitalCommons@University of Nebraska - Lincoln. 


\title{
Do physicians examine patients in contact isolation less frequently? A brief report
}

\author{
Sanjay Saint, MD, MPH, ${ }^{\mathrm{a}, \mathrm{b}, \mathrm{d}}$ Leigh Ann Higgins, MD, ${ }^{\mathrm{c}}$ Brahmajee K. Nallamothu, MD, MPH, ${ }^{\mathrm{b}}$ and Carol Chenoweth, $\mathrm{MD}^{\mathrm{b}}$ \\ Ann Arbor, Michigan, and Richmond, Virginia
}

\begin{abstract}
Background: Patients who are hospitalized and infected with multidrug-resistant bacteria are usually placed in contact isolation, which requires hospital personnel to gown and glove before patient examination. Contact isolation with active culture surveillance appears beneficial in preventing the spread of drug-resistant infections; however, contact isolation may impede the ability to examine patients as a result of the additional effort required to gown and glove. We assessed whether patients who are hospitalized and placed under contact precautions are examined less often by second- and third-year medical residents (ie, senior medical residents), and attending physicians during morning rounds.
\end{abstract}

Method: We conducted a prospective cohort study on the inpatient medical services at 2 university-affiliated medical centers. We directly observed senior medical residents and attending physicians during morning rounds, and recorded the contact precaution status of the patient and whether they were examined by either physician.

\begin{abstract}
Results: Of a total of 139 patients, 31 (22\%) were in contact isolation. Senior medical residents examined 26 of 31 patients $(84 \%)$ in contact isolation versus 94 of 108 patients $(87 \%$ ) not in contact isolation (relative risk, 0.96; 95\% confidence interval, 0.81-1.14; $P=.58)$. In comparison, attending physicians examined 11 of 31 patients $(35 \%)$ in contact isolation versus 79 of 108 patients $(73 \%)$ not in contact isolation (relative risk, $0.49 ; 95 \%$ confidence interval, $0.30-0.79 ; P<.001$ ).
\end{abstract}

Discussion: Attending physicians are about half as likely to examine patients in contact isolation compared with patients not in contact isolation. (Am J Infect Control 2003;31:354-6.)

Antimicrobial-resistant bacteria, such as vancomycinresistant enterococci (VRE), have increased dramatically in hospitals around the United States. To curb the spread of such organisms, the Hospital Infection Control Practices Advisory Committee of the Centers for Disease Control and Prevention has recommended that contact precautions be used for all patients who are hospitalized, and colonized or infected with VRE or with other antimicrobial-resistant organisms, such methicillinresistant Staphylococcus aureus. ${ }^{1,2}$ Contact precautions require physicians to wear gowns and gloves before patient examination. Although contact isolation coupled

From the Ann Arbor Department of Veterans Affairs Medical Centera; Departments of Internal Medicine, University of Michigan ${ }^{b}$ and Virginia Commonwealth Universityc; and Patient Safety Enhancement Program, University of Michigan Health System. ${ }^{d}$

Supported by a career development award from the Health Services Research and Development Program of the Department of Veterans Affairs and a Patient Safety Developmental Center Grant from the Agency for Healthcare Research and Quality (P20-HSI I540) (Dr Saint).

Presented, in part, at the National Association of Inpatient Physicians 4th Annual National Meeting, Atlanta, GA, March 2001.

Reprint requests: Sanjay Saint, MD, MPH, Division of General Medicine, University of Michigan Health System, Room 7E08, 300 NIB, Campus Box 0429, Ann Arbor, MI 48109-0429.

$0196-6553 / 2003 / \$ 30.00+0$

doi: $10.1067 / \mathrm{mic} .2003 .50$ with active culture surveillance appears beneficial in preventing the spread of drug-resistant infections, ${ }^{3-5}$ contact isolation is commonly used without active culture surveillance where the benefits of this policy are less clear. Unfortunately, the use of contact isolation may have adverse effects, such as impeding a physician's ability to examine patients. We, thus, assessed whether patients who are hospitalized and placed in contact isolation are examined less often by senior medical residents and attending physicians during morning rounds compared with patients not in contact isolation.

\section{METHODS}

A prospective cohort study was conducted at 2 university-affiliated medical centers from October 1999 to March 2000. The study participants were senior medical residents (ie, second- and third-year residents in internal medicine), and attending physicians who were rotating on the general medicine and subspecialty services at the 2 medical centers. On several days per month during the study period (depending on the availability of the investigator), one of the study investigators (L.A.H.) accompanied a medical team during morning rounds recording: (1) the contact precaution status of each patient; and (2) whether or not patients were physically examined by a senior resident or attending physician. Morning rounds were chosen because this is the time of day when the study participants (ie, senior 

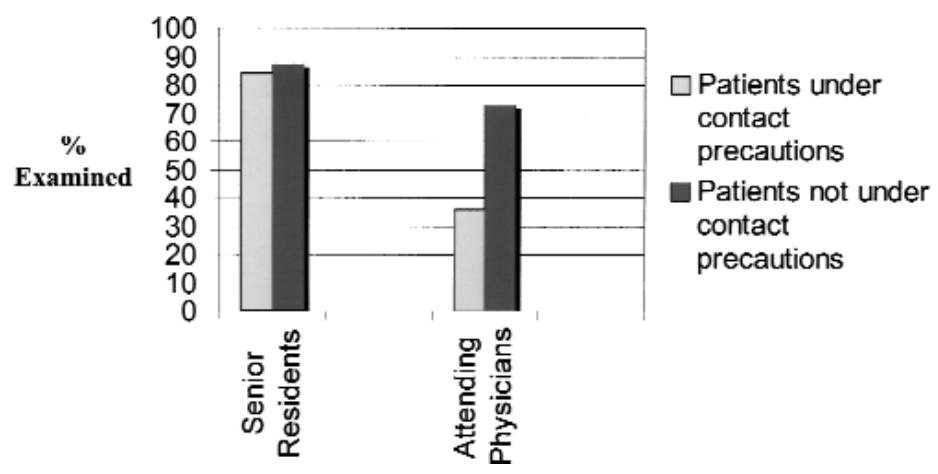

Fig I. Percent of patients examined by senior residents or attending physicians by contact precaution status.

medical resident, attending physician) would be most likely to examine patients as management decisions are made during this time period. Given the antimicrobialresistance patterns and practices at the 2 study hospitals, the vast majority of the patients under contact precautions were either infected or colonized with VRE; however, a few patients may have been infected or colonized with other resistant organisms such as methicillinresistant Staphylococcus aureus. "Physically examined" was defined as examining (by auscultation, palpation, or percussion) at least one organ system. Residents and attending physicians were unaware of why they were being observed. All data were entered anonymously and, thus, could not be linked to any given physician. Because all data were anonymous, the study protocol was reviewed and considered exempt by the institutional review board.

To assess for differences in the percentage of patients examined by a senior resident or attending physician on the basis of contact precaution status, $\chi^{2}$ tests were used. In addition, the relative risk of a patient being examined given his or her contact precaution status was calculated along with appropriate $95 \%$ confidence intervals. $P$ values $<.05$ were considered statistically significant. All SE were adjusted using robust variance estimates to account for possible clustering at the medical-team level.

\section{RESULTS}

A total of 139 patients were evaluated, 31 (22\%) of whom were in contact isolation. The percentage of patients examined differed by physician training level (Fig 1). Senior medical residents examined 26 of 31 patients $(84 \%)$ under contact precautions versus 94 of 108 patients $(87 \%)$ not under contact precautions (relative risk of being examined $=0.96 ; 95 \%$ confidence interval $=0.81-1.14 ; P=.58)$. Attending physicians, in contrast, examined 11 of 31 patients $(35 \%)$ under contact precautions versus 79 of 108 patients (73\%) not under contact precautions (relative risk $=0.49 ; 95 \%$ confidence interval $=0.30-0.79 ; P<.001)$.

\section{DISCUSSION}

Our study has 2 important findings. First, attending physicians were about half as likely to examine patients under contact precautions compared with patients not under contact precautions. Almost twothirds of patients in contact isolation were not examined during morning rounds by the attending physician. Second, resident physicians did not alter their examination behavior on the basis of contact precaution status of the patient.

The use of contact precautions in epidemic or outbreak situations appears to decrease the transmission of VRE. Boyce et $\mathrm{al}^{6}$ report that during single-strain VRE outbreaks, new cases continued to occur with the use of private rooms and gloves. Control of the outbreak did not occur until use of gowns was added to the control measures. ${ }^{6}$ At a different institution, a hospitalwide outbreak of VRE was controlled through the use of weekly screening cultures with contact isolation of patients who were colonized. ${ }^{7}$ Finally, a large recent study found that surveillance cultures and the use of contact precautions for patients who were colonized was effective in reducing the transmission of VRE in health care facilities over the Siouxland region of Iowa, Nebraska, and South Dakota. ${ }^{8}$

Data supporting the use of contact isolation in hospitals with endemic VRE are mixed, even though contact precautions are widely recommended ${ }^{1,2}$ and commonly used in these settings. Universal use of gowns and gloves did not prevent the acquisition of VRE in a medical intensive care unit with endemic VRE compared with the universal use of gloves alone. ${ }^{9}$ In the gown and glove cohort, $25.8 \%$ of patients acquired VRE in the unit, whereas $23.9 \%$ of patients in the glove-only group acquired VRE. ${ }^{9}$ On the other hand, a study conducted in an adult oncology inpatient unit found that contact isolation may be useful. ${ }^{10}$ This investigation found that when contact precau- 
tions were used in addition to other infection control strategies to control endemic VRE (such as reducing vancomycin use, and active inpatient and environmental surveillance) VRE colonization decreased by approximately $50 \%(P<.001) .{ }^{10}$ Recent studies indicate that the use of contact isolation with active culture surveillance appears to be both effective and economically attractive. ${ }^{3-5}$

Although the efficacy of contact isolation without the use of active culture surveillance in settings in which VRE are endemic is unclear, the costs associated with this practice have recently been highlighted. From a hospital's perspective, the universal and routine use of gowns and gloves, and patient cohorting are associated with substantial expense. Kirkland and Weinstein ${ }^{11}$ estimate that the incremental cost of placing a patient who is critically ill in contact isolation is $\$ 1627$ per patient in their hospital. Some observers even argue that isolating patients because of their status as carriers of antimicrobial-resistant infection can lead to negative psychologic effects, such as depression and anxiety. ${ }^{12}$ Finally, we have observed that even when patients under contact isolation are physically examined by their attending physician, the examination may be limited by the use of the often suboptimal stethoscope dedicated to each patient who is infected. However, because the costs of antimicrobial-resistant infections are substantial, any intervention that curbs the spread of resistance may be economically attractive despite the initial costs. ${ }^{3,5}$ Thus, additional research is needed to compare the efficacy and costs of various infection control methods for preventing the spread of antimicrobial-resistant infections in patients who are hospitalized. ${ }^{13}$

Our study should be interpreted in the context of the following limitations. First, we did not collect data on severity-of-illness or on comorbid conditions and, thus, could not adjust for these factors. However, patients with multidrug-resistant bacteria are generally quite ill and may have more complicated problems than patients not in contact isolation. Thus, the deliberate decision of attending physicians to not examine patients under contact precautions because they are healthier than patients not under contact precautions seems unlikely. Second, the appropriateness of whether or not a patient should be examined was not determined. Physical examination, however, is an integral part of the physician-patient relationship and one that should not be systematically undermined by hospital policy. Third, we did not assess whether patient care or clinical outcomes differed on the basis of physical examination. Fourth, we could not account for physicians who may have gone back later in the day to examine their patients under contact precautions. Although this is a theoretic possibility, we doubt that it happened frequently enough to alter our main results.

Finally, we did not evaluate the habits of other health care providers, such as nurses, who may also be affect- ed by a patient's contact precaution status. A recent study has found that physician and nonphysician health care workers were about 2 times less likely to enter the rooms of patients in contact isolation. ${ }^{11}$ This study, conducted in the medical intensive care unit at Duke University Medical Center, reported that health care providers had a mean of 2.1 contacts/h with patients in contact isolation compared with 4.2 contacts/h with patients not in contact isolation $(P=.03) .{ }^{11}$

In conclusion, our prospective study reveals that patients placed under contact precautions are about half as likely to be examined by their attending physicians compared with patients not in contact isolation. Fortunately, we observed no change in examination rates among resident physicians on the basis of a patient's contact isolation status.

\section{References}

I. Hospital Infection Control Practices Advisory Committee (HICPAC). Recommendations for preventing the spread of vancomycin resistance. Am J Infect Control 1995;23:87-94.

2. Garner JS. Guideline for isolation precautions in hospitals: the hospital infection control practices advisory committee [published erratum, Infect Control Hosp Epidemiol 1996;17:214]. Infect Control Hosp Epidemiol 1996; 17:53-80.

3. Mayhall CG. Control of vancomycin-resistant enterococci: it is important, it is possible, and it is cost-effective. Infect Control Hosp Epidemiol 2002;23:420-3.

4. Srinivasan A, Song XY, Ross T, Merz W, Brower R, Perl TM. A prospective study to determine whether cover gowns in addition to gloves decrease nosocomial transmission of vancomycin-resistant enterococci in an intensive care unit. Infect Control Hosp Epidemiol 2002;23:424-8.

5. Muto CA, Giannetta ET, Durbin LJ, Simonton BM, Farr BM. Costeffectiveness of perirectal surveillance cultures for controlling vancomycin-resistant Enterococcus. Infect Control Hosp Epidemiol 2002;23:429-35.

6. Boyce JM, Mermel LA, Zervos MJ, Rice LB, Potter-Bynoe G, Giorgio $C$, et al. Controlling vancomycin-resistant enterococci [published erratum, Infect Control Hosp Epidemiol 1996;17:2II] [comments]. Infect Control Hosp Epidemiol 1995; 16:634-7.

7. Byers KE,Anglim AM,Anneski CJ, Germanson TP, Gold HS, Durbin LJ, et al. A hospital epidemic of vancomycin-resistant enterococcus: risk factors and control. Infect Control Hosp Epidemiol 200I;22:140-7.

8. Ostrowsky BE, Trick WE, Sohn AH, Quirk SB, Holt S, Carson LA, et al. Control of vancomycin-resistant enterococcus in health care facilities in a region. N Engl J Med 200I;344:1427-33.

9. Slaughter S, Hayden MK, Nathan C, Hu TC, Rice T,Van Voorhis J, et al.A comparison of the effect of universal use of gloves and gowns with that of glove use alone on acquisition of vancomycin-resistant enterococci in a medical intensive care unit. Ann Intern Med 1996; I 25:448-56.

10. Montecalvo MA, Jarvis WR, Uman J, Shay DK, Petrullo C, Rodney K, et al. Infection-control measures reduce transmission of vancomycin-resistant enterococci in an endemic setting. Ann Intern Med 1999;131:269-72.

II. Kirkland KB, Weinstein JM. Adverse effects of contact isolation [letter]. Lancet 1999;354:I I77-8.

12. Peel RK, Stolarek I, Elder AT. Is it time to stop searching for MRSA? Isolating patients with MRSA can have long term implications. BMJ 1997;315:58.

13. Saint S,Atherton S, Lipsky BA, McDonald L, Strausbaugh L. Controlling the spread of vancomycin-resistant enterococci with contact precautions: time for a randomized trial. Int J Infect Dis 1999;3:179-80. 